\title{
Study Objective Description
}

National Cancer Institute

\section{Source}

National Cancer Institute. Study Objective Description. NCI Thesaurus. Code C94090.

The textual representation of the study objective. 